\title{
Comportamiento bajo cargas estáticas de sistemas provisionales de protección de borde realizados con elementos de acero
}

\author{
Behavior under static loads of temporary edge protection \\ systems built with steel elements
}

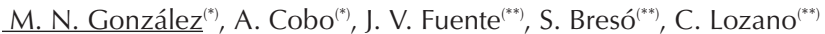

\section{RESUMEN}

La norma UNE-EN 13374 "Sistemas provisionales de protección de borde. Especificaciones del producto, métodos de ensayo" $^{\prime \prime}$ (1) clasifica los sistemas provisionales de protección de borde (SPPB) en tres clases (A, B y C), en función del ángulo de la superficie de trabajo y de la altura de caída de la persona a proteger. Los sistemas clase A son los indicados cuando la inclinación de la superficie de trabajo es menor de $10^{\circ}$. La norma establece los requisitos de flecha y de resistencia de los SPPB. Los requisitos se pueden comprobar tanto analítica como experimentalmente.

El objetivo del trabajo ha sido la evaluación del comportamiento de los SPPB utilizados habitualmente en las obras y establecer los cambios necesarios para que cumplan con la norma UNE-EN 13374. Para ello se han evaluado analítica y experimentalmente tres SPPB clase A, fabricados con acero S235.

Los resultados obtenidos muestran que, el sistema empleado de forma habitual en obras no supera los requisitos de la norma ni analítica ni experimentalmente. El tercer sistema supera los requisitos con las dos metodologías de análisis. El segundo sistema supera los requisitos cuando la evaluación se realiza analíticamente pero no cuando la vía utilizada es la experimental.

754-3

Palabras clave: Prevención, Barandillas de acero, Métodos de evaluación, Protección de caída, Obras de construcción.

\section{SUMMARY}

The standard UNE-EN 13374 "Temporary edge protection systems. Product specification, test methods" (1) classifies the temporary edge protection systems (TEPS) under three classes ( $A, B$ and $C$ ), based on the angle of the surface of work and the fall of the person to protect. The class A systems are the indicated when the inclination of the work surface is smaller than $10^{\circ}$. The standard establishes the requirements of deflection and strength of the TEPS. The requirements can be verified both analytically as experimentally.

The aim of this work has been the evaluation of the behaviour of three TEPS class A -those commonly used in the construction worksand to establish the necessary changes so that they comply with the standard UNE-EN 13374. For it, three TEPS class A, made of steel S235, have been evaluated analytically and experimentally.

The results obtained show that the system normally used in works does not comply with the requirements of the standard neither analytically nor experimentally. The third system fulfils the requirements with both methods of analysis. The second system surpasses the requirements when the evaluation is carried out analytically but not when it is performed experimentally.

Keywords: safety, steel guardrails, evaluation methods, fall protection, construction sites. 


\section{INTRODUCCIÓN}

Los accidentes en construcción debidos a la caída en altura suponen un alto porcentaje del total de los ocurridos en el sector (2-4).

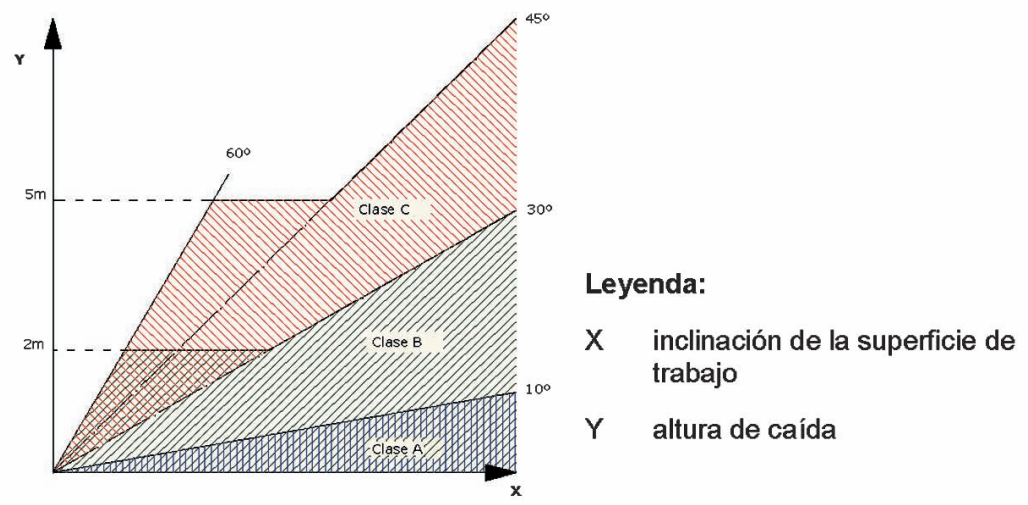

Las normas y reglamentos, tanto a nivel nacional como a nivel internacional, limitan la altura de caída (5-8).

La estrategia preventiva frente al riesgo de caída en altura, establece el siguiente orden de actuación: en primer lugar eliminar los riesgos en el origen; si no es posible, la altura de caída debe ser limitada mediante protecciones colectivas; en último lugar hay que dotar a los trabajadores de equipos de protección individual frente a la caída $(9,10)$.

La eliminación de los riesgos en el origen se puede conseguir planificando la ejecución de la obra, ejecutando la obra con medidas de protección integradas en la propia estructura o instalando protecciones colectivas que impidan la caída.

Los sistemas que limitan la altura de caída suelen estar formados por redes que transmiten la energía del impacto a la estructura en construcción, a través de elementos más rígidos, generalmente metálicos. En Europa gran parte de estos sistemas están normalizados (11, 12). Algunas tipologías como las redes sobre pescante, se vienen empleando en España desde hace decenios. Sin embargo, aspectos esenciales acerca de su comportamiento como la máxima aceleración experimentada por el cuerpo después del impacto sobre la red, no se ha conocido hasta la realización de trabajos recientes $(13,14)$.

La utilización de equipos de protección individual supone generalmente la realización de anclajes a la estructura. En este caso es necesario conocer la resistencia al arrancamiento del anclaje en el punto donde se amarra (15-17).
La protección frente a caída en altura mediante sistemas provisionales de protección de borde (SPPB) constituye un sistema eficaz porque elimina el riesgo en el origen, impidiendo la caída y evitando por tanto la posibilidad de sufrir lesiones cuando un trabajador impacta contra otro sistema que solo limita la altura de caída.

Los documentos consultados que regulan los SPPB (18-20) exigen la superación, por parte de éstos, de requisitos de tipo geométrico y de tipo mecánico. Los requisitos de tipo geométrico establecen las dimensiones de los SPPB para que el trabajador no rebase el sistema y caiga o los objetos no pasen desde el forjado al vacío. Los requisitos de tipo mecánico exigen una determinada resistencia al sistema y limitan sus desplazamientos frente a unas cargas dadas.

Teniendo en cuenta que sólo se deben emplear sistemas de protección colectiva de cuya resistencia y comportamiento se tengan garantías, es prioritario utilizar sistemas que cumplan los requisitos establecidos en las distintas normas UNE, elaboradas para cumplir una serie de especificaciones técnicas que determinan la idoneidad del producto. En relación a la prevención de caídas a distinto nivel en obras de construcción, los requisitos establecidos por la normativa española han sido muy genéricos hasta que, afortunadamente, a finales del año 2004, se produce un punto de inflexión. La norma UNE-EN 13374 específica los requisitos de comportamiento y métodos de ensayo para los SPPB empleados durante la construcción o el mantenimiento de edificios y otras estructuras.

La norma clasifica los SPPB en tres clases (A, B y C), en función de la inclinación de la superficie de trabajo y de la altura de caída de la persona a proteger (Figura 1). La norma indica los requisitos, en términos geométricos y mecánicos, que deben superar los SPPB en función de la clase a la que pertenecen.

Los sistemas clase A, que son los más empleados, sólo se pueden utilizar cuando el ángulo de inclinación de la superficie de trabajo es menor de $10^{\circ}(1)$.

Lamentablemente, a día de hoy, la inmensa mayoría de los SPPB utilizados en obras no han sido objeto de una evaluación previa y no se conoce su comportamiento mecánico.

\section{TÉCNICAS DE EVALUACIÓN DE SPPB CLASE A}

\subsection{Tipos de análisis}

Para verificar los requisitos mecánicos que deben cumplir los SPPB, la norma UNE-EN 
13374 permite realizar su evaluación tanto analítica como experimentalmente.

\subsection{Procedimiento analítico}

Según la norma, el cálculo debe efectuarse de acuerdo con el método de los Estados Límites, empleando las normas europeas para ingeniería de las estructuras. En el caso de elementos de acero, la norma de referencia es ENV 1993-1-1 - Eurocódigo 3 (21).

Se analizan tres situaciones: Estado Límite Último (ELU), Estado Límite de Servicio (ELS) y carga accidental.

En ELU se comprueba que cada protección de borde y cada uno de sus componentes, excepto los plintos, deben estar diseñados para resistir una carga $\mathrm{F}_{\mathrm{H} 1}=0,30 \mathrm{kN}$ aplicada perpendicularmente al eje del poste. Los plintos deben soportar una carga $\mathrm{F}_{\mathrm{H} 2}=0,20 \mathrm{kN}$. Estas cargas deben aplicarse en los puntos más desfavorables.

Para la evaluación frente a este estado límite, debe emplearse un coeficiente de mayoración de acciones $\gamma_{\mathrm{F}}$ de valor 1,5 y un coeficiente de minoración de la resistencia del material $\gamma_{\mathrm{M}}$ de valor 1,1.

Para superar el ELS, la flecha no debe ser mayor de $55 \mathrm{~mm}$. La flecha especificada se define como la flecha de todo el sistema al que se aplica la fuerza $F_{\mathrm{T} 1}$ de $0,30 \mathrm{kN}$. En el caso del rodapié, la fuerza a aplicar $\mathrm{F}_{\mathrm{T} 2}$ toma el valor de $0,20 \mathrm{kN}$.

En cuanto al estudio de las cargas accidentales, se indica que la barandilla principal, la barandilla intermedia y el plinto, deben resistir una carga puntual gravitatoria $F_{D}=1,25 \mathrm{kN}$. Esta carga debe aplicarse en la posición más desfavorable del SPPB, dentro de un sector inclinado $\pm 10^{\circ}$ respecto de la vertical.

Para la evaluación frente a ELS y cargas accidentales los coeficientes de mayoración de acciones y de minoración de la resistencia de los materiales toman el valor unidad.

\subsection{Procedimiento experimental}

La comprobación en ELS y en ELU se realiza aplicando acciones horizontales según el ciclo de carga que se expone a continuación. Se aplica una carga inicial de $0,10 \mathrm{kN}$ al sistema. Esta carga se mantiene durante un minuto y, a continuación se descarga el sistema, quedando éste con un desplazamiento residual que constituye la flecha de referencia, $\delta_{1}$. A continuación se aplica la carga del ensayo correspondiente, se mantiene un minuto $y$ se descarga.

\section{Comprobación en ELS}

La carga máxima para este ensayo, $\mathrm{Q}_{\mathrm{K}}$, vale $0,30 \mathrm{kN}$ para las barandillas y el poste $\mathrm{y}$ 0,20 kN para el rodapié, aplicada en cinco incrementos regulares. Una vez alcanzada la carga, debe mantenerse durante un minuto con el objeto de determinar las características de fluencia del sistema.

El máximo desplazamiento horizontal experimentado por el sistema, constituye la flecha del mismo y no debe superar el valor de $55 \mathrm{~mm}$.

\section{Comprobación en ELU}

La carga máxima de ensayo $F_{\text {máx. }}$ se obtiene como $F_{\text {máx. }}=\gamma_{F} \cdot \gamma_{M} \cdot Q_{K}$, donde $\gamma_{F}$ y $\gamma_{M}$ son los coeficientes parciales de seguridad para ELU (mayoración de acciones y minoración de resistencia del material respectivamente) y $Q_{K}$ es la carga característica según el elemento considerado. Debe aplicarse en diez incrementos regulares y mantenerse durante un minuto. Debe medirse la flecha de la protección de borde, $\delta_{\text {máx }}$ bajo la carga máxima.

La carga de ensayo debe retirarse y medirse la flecha residual, $\delta_{\text {res }}$. A continuación el sistema debe ser cargado con un esquema de cargas idéntico, incrementándose hasta la carga de rotura, $R_{u}$ que provoca un fallo notable a nivel del conjunto del sistema o en uno de los elementos que lo componen.

Se debe registrar la flecha en la posición de referencia $\delta_{1}$, la flecha bajo la carga máxima $\delta_{\text {máx }}$ la flecha residual $\delta_{\text {res }}$ y la carga de rotura, $R_{u}$.

El ensayo se considera válido cuando se cumplen simultáneamente las tres condiciones siguientes: bajo la carga máxima no se producen plastificaciones o roturas, la flecha residual es inferior al $10 \%$ de la flecha bajo la carga máxima, y $R_{u}$ es superior a 1,2 veces la máxima carga de ensayo.

\section{Comprobación para carga accidental}

Se debe aplicar en el punto más desfavorable del SPPB una carga vertical descendente de 1,25 kN y comprobar que el sistema es capaz de resistirla.

\section{TRABAJO EXPERIMENTAL REALIZADO}

\subsection{Características de los sistemas estudiados}

El objetivo planteado con la realización de este trabajo ha sido la evaluación del comportamiento de los SPPB clase A utilizados 
2. Características geométricas del sistema 1.

3. Modelo de cálculo para las barandillas y el poste. habitualmente en obras. Para ello se ha utilizado como referencia la norma UNE-EN 13374. alojar tanto los postes de sección cuadrada de $35 \mathrm{~mm}$ de lado, como los postes de sección circular de $40 \mathrm{~mm}$ de diámetro. En la

Tabla 1

Características geométricas de los tres sistemas ensayados.

\begin{tabular}{|c|c|c|c|}
\hline & Sistema $1(\mathrm{~S} 1)$ & Sistema $2(\mathrm{~S} 2)$ & Sistema $3(\mathrm{~S} 3)$ \\
\hline BARANDILLAS & $\circ 25 \cdot 1,5 \mathrm{~mm}$ & $\circ 40 \cdot 1,5 \mathrm{~mm}$ & $\circ 40 \cdot 2 \mathrm{~mm}$ \\
\hline POSTES VERTICALES & $\circ 40 \cdot 1,5 \mathrm{~mm}$ & $\square 35 \cdot 1,5 \mathrm{~mm}$ & $\circ 40 \cdot 2 \mathrm{~mm}$ \\
\hline RODAPIÉ & \multicolumn{2}{|c|}{ telescópico, fabricado en chapa conformada } \\
\hline
\end{tabular}

Se han analizado tres SPPB con una luz entre postes de $2.400 \mathrm{~mm}$ y una altura de 1.000 $\mathrm{mm}$, medida desde el nivel de referencia hasta el borde superior de la barandilla principal. Las barandillas principal e intermedia y el poste se han resuelto con secciones tubulares de acero S235. Las orejetas de los postes son de acero S275. El rodapié, telescópico, ha sido fabricado en acero conformado en frío.

En la Tabla 1 se detallan las características geométricas de los tres sistemas.
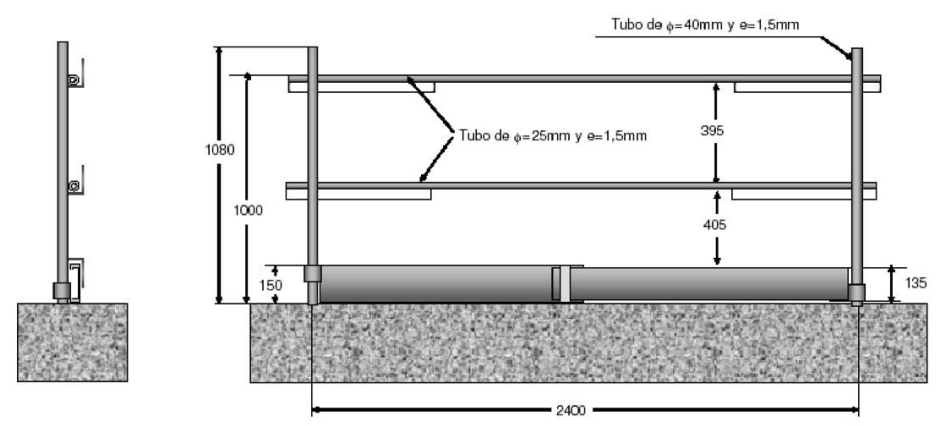

Cotas en $\mathrm{mm}$
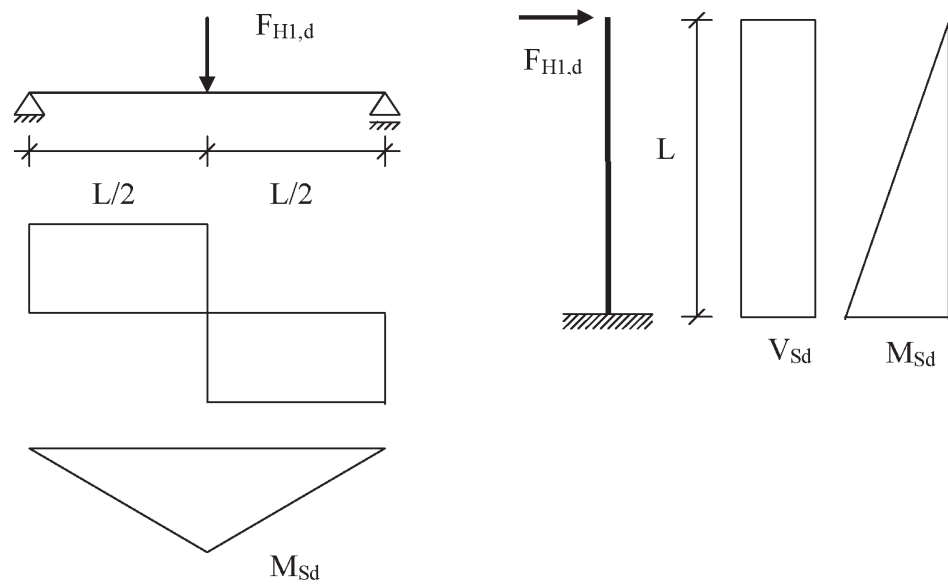

Los tres sistemas se han anclado a una viga de hormigón armado en la que se han embebido cartuchos de PVC preparados para
Figura 2 se muestra, a modo de ejemplo, la disposición y las características geométricas del sistema 1.

Las dimensiones de uno de los sistemas estudiados son las empleadas habitualmente para la protección de bordes de forjado en obras de construcción. El segundo de los sistemas se utiliza en ocasiones excepcionales. Las dimensiones del tercer sistema se han determinado después de realizar un cálculo en base a la norma UNE-EN 13374.

\subsection{Procedimiento de análisis algebraico}

En la evaluación analítica se han adoptado los siguientes modelos de cálculo: las barandillas se han considerado como vigas biapoyadas, siendo los apoyos los vínculos con el poste. El poste se ha considerado como una ménsula, empotrado en el forjado, como se comprobará más adelante esta hipótesis queda del lado de la inseguridad en el cálculo de flechas al no considerar los movimientos producidos por la deformación del cartucho de plástico.

Para el cálculo del sistema, se han estudiado los elementos por separado, incorporando en el análisis de cada uno de ellos los efectos producidos por el resto.

El análisis en ELU es idéntico para la barandilla principal e intermedia. La situación más desfavorable para estos elementos se produce cuando la carga está situada en el centro de la barandilla, resultando el máximo momento flector en la barra.

En el poste, la situación más desfavorable se produce cuando la carga se aplica en su extremo volado y la sección más desfavorable es la base, donde se produce el máximo momento flector y el máximo cortante (Figura 3).

Para el cálculo del sistema en ELS el movimiento horizontal del sistema se ha obtenido como la suma de la flecha de la barandilla 
cargada en el centro de la luz y la flecha del poste. La flecha en el poste se ha calculado con una acción que es la mitad de la carga de la barandilla y aplicada en su extremo (Figura 4). Se ha comprobado la flecha en la barandilla principal, que es mayor que la flecha de la barandilla intermedia.

El cálculo para acciones accidentales en la barandilla sigue la misma metodología que el cálculo en ELU aplicando una carga vertical de 1,25 kN en la posición más desfavorable y utilizando como coeficientes de mayoración de acciones o minoración de la resistencia de los materiales el valor unidad.

\subsection{Procedimiento de análisis experimental}

Todas las pruebas se han Ilevado a cabo en las instalaciones del Laboratorio de Elementos de Seguridad del Instituto Tecnológico de la Construcción (AIDICO). Se ha utilizado un pórtico de ensayos dotado de dos actuadores de carga, uno para la aplicación de cargas horizontales y otro para la aplicación de cargas verticales. Los movimientos se han obtenido con un transductor de desplazamiento. Un sistema de control y adquisición de datos a través de software específico ha registrado los datos de carga y desplazamiento en cada uno de los ensayos.

En cada uno de los SPPB ensayados, los ciclos de carga se aplican en los puntos más desfavorables del sistema, elegidos bajo criterios normativos y del propio equipo investigador (Figura 5).

La carga aplicada en los puntos 1, 2 y 3, situados en el centro de las barandillas superior e inferior y del rodapié, produce el máximo momento flector en estos elementos (requisitos de resistencia y carga accidental) y el
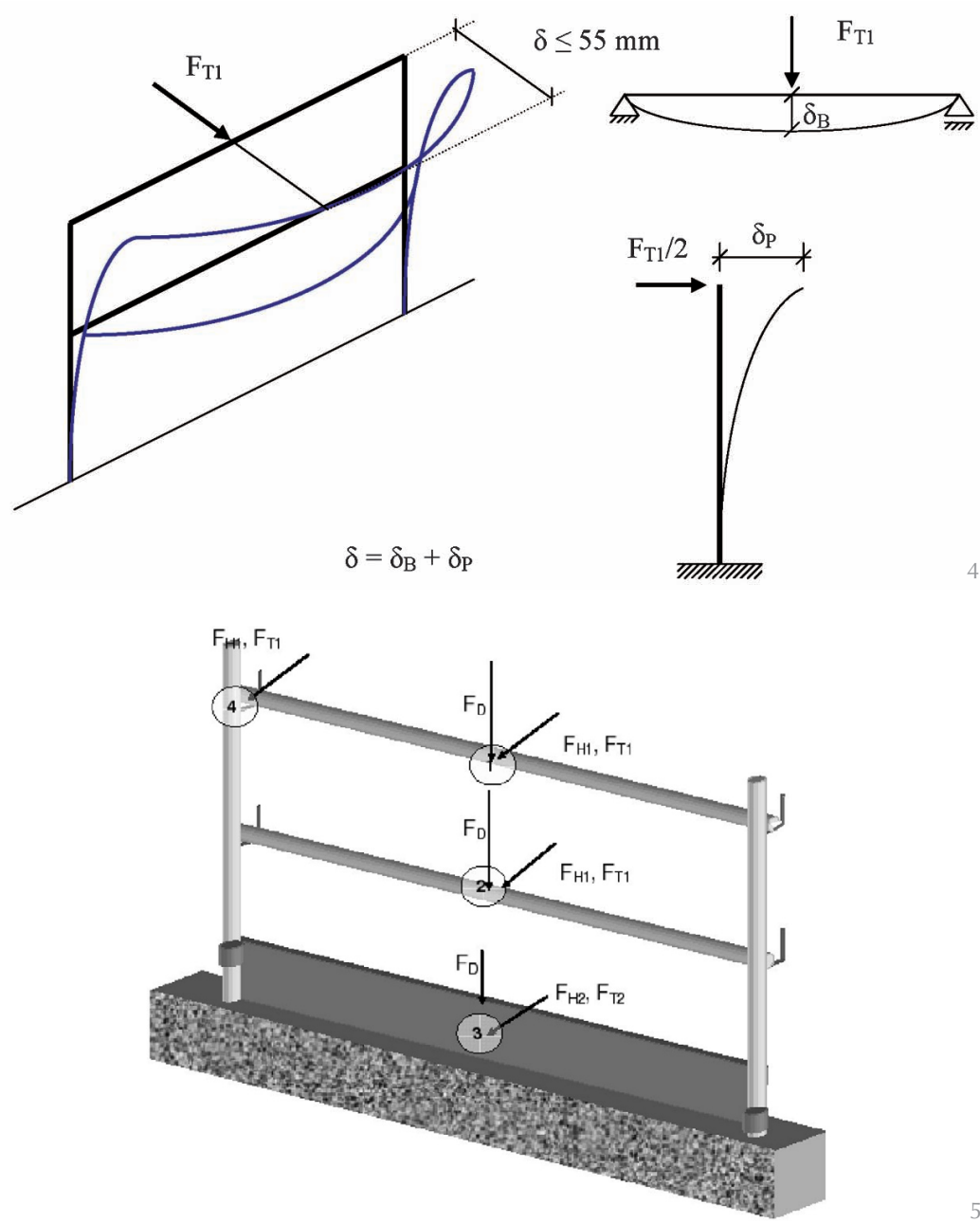

máximo desplazamiento del sistema (requisito de flecha). El punto 4, situado en el extremo del poste, produce el máximo momento flector y el máximo desplazamiento en el poste.

En SPPB formados por tubos metálicos no es necesario comprobar la sección próxima al

Tabla 2

Resultados de los ensayos de flecha y resistencia.

\begin{tabular}{|c|c|c|c|c|c|c|c|}
\hline \multirow{3}{*}{ ELEMENTO } & \multirow{3}{*}{ SISTEMA } & \multicolumn{6}{|c|}{ ENSAYO } \\
\hline & & \multicolumn{2}{|c|}{ Flecha } & \multicolumn{4}{|c|}{ Resistencia } \\
\hline & & $\begin{array}{l}\mathrm{F}_{\mathrm{T} 1} \\
(\mathrm{kN})\end{array}$ & $\begin{array}{c}\delta \\
(\mathrm{mm})\end{array}$ & $\begin{array}{l}\mathrm{F}_{\mathrm{H} 1} \\
(\mathrm{kN})\end{array}$ & $\begin{array}{c}\mathrm{R}_{\mathrm{u}} \\
(\mathrm{kN})\end{array}$ & $\begin{array}{l}\delta_{\text {máx }} \\
(\mathrm{mm})\end{array}$ & $\begin{array}{c}\delta_{\text {res }} \\
(\mathrm{mm})\end{array}$ \\
\hline \multirow{3}{*}{$\begin{array}{l}\text { Barandilla } \\
\text { principal }\end{array}$} & S1 & 0,30 & 66,67 & 0,50 & 0,57 & 138,24 & 30,27 \\
\hline & S2 & 0,30 & 28,87 & 0,50 & 1,34 & 46,69 & 3,17 \\
\hline & S3 & 0,30 & 21,66 & 0,50 & 1,94 & 37,20 & 1,20 \\
\hline \multirow{3}{*}{ Poste } & S1 & 0,30 & 20,98 & 0,50 & 1,08 & 28,62 & 1,60 \\
\hline & S2 & 0,30 & 19,48 & 0,50 & 0,82 & 34,12 & 6,25 \\
\hline & S3 & 0,30 & 14,97 & 0,50 & 1,41 & 23,15 & 1,32 \\
\hline Rodapié & $\mathrm{S} 1, \mathrm{~S} 2, \mathrm{~S} 3$ & 0,20 & 13,43 & 0,35 & 0,63 & 30,18 & 2,25 \\
\hline
\end{tabular}

4. Obtención de la flecha del sistema.

5. Disposición del ensayo de SPPB según la norma UNE-EN 13374

$\mathrm{F}_{\mathrm{T} 1}$ : Fuerza aplicada para cumplir requisito de flecha (aplicada en puntos $1,2$ y 4$)$.

$\mathrm{F}_{\mathrm{T} 2}$ : Fuerza aplicada para cumplir requisito de flecha (aplicada en punto 3).

$\mathrm{FH}_{1}$ : Fuerza aplicada para cumplir requisito de resistencia (aplicada en puntos 1,2 y 4 ).

$\mathrm{FH}_{2}$ : Fuerza aplicada para cumplir requisito de resistencia (aplicada en punto 3).

$\mathrm{F}_{\mathrm{D}}$ : Carga Accidental (vertical). 
6. Resultados del ensayo de flecha sobre el punto central de la barandilla principal. sobre el punto superior del poste. tencia sobre el punto central de la barandilla principal.
Resultados del ensayo de flecha

Resultados del ensayo de resis-

apoyo en el poste de los elementos horizontales. La aplicación de una carga en dicha sección produciría el máximo cortante, de aproximadamente el valor de la carga, pero la comprobación a flexión resulta infinitamente más desfavorable.

\section{RESULTADOS OBTENIDOS}

En la Tabla 2 se muestran los resultados obtenidos en los ensayos de flecha y resistencia

Ensayo de flecha barandilla principal

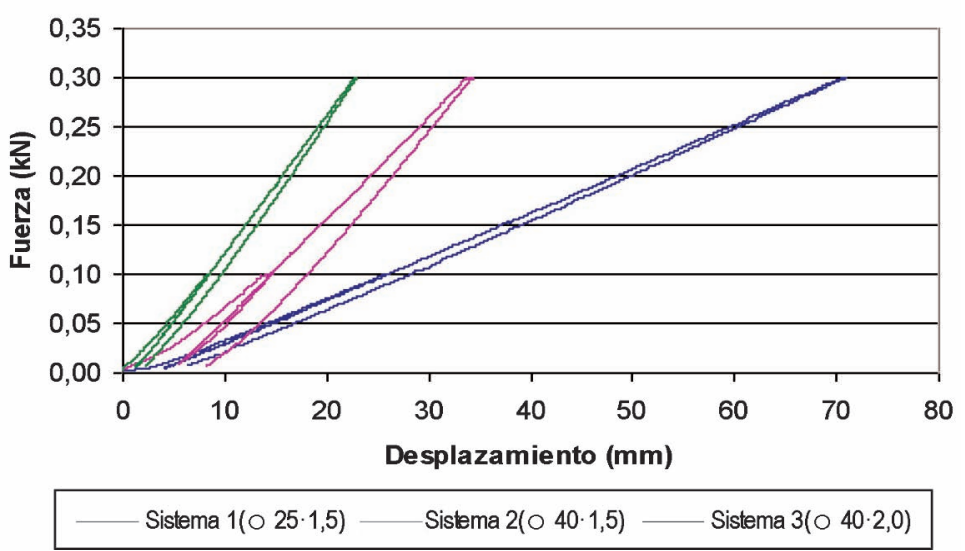

Ensayo de flecha postes de acero

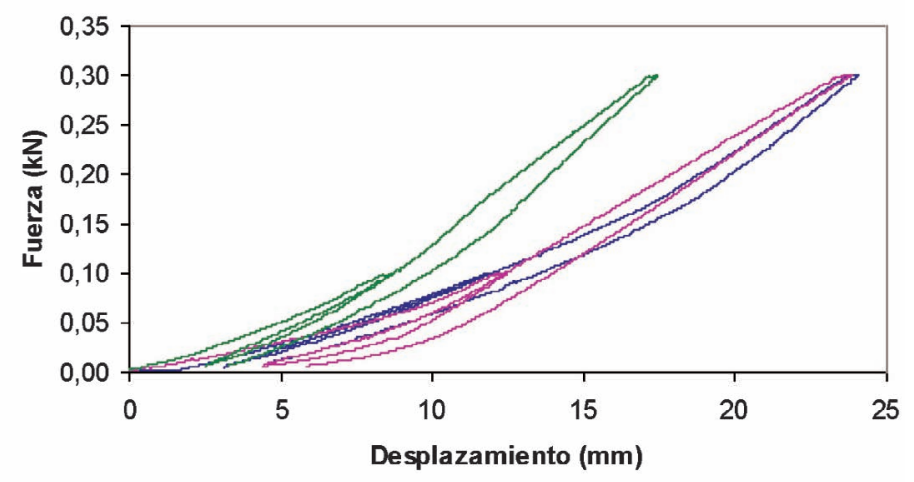

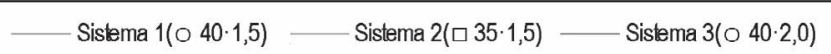

Ensayo de resistencia barandilla principal

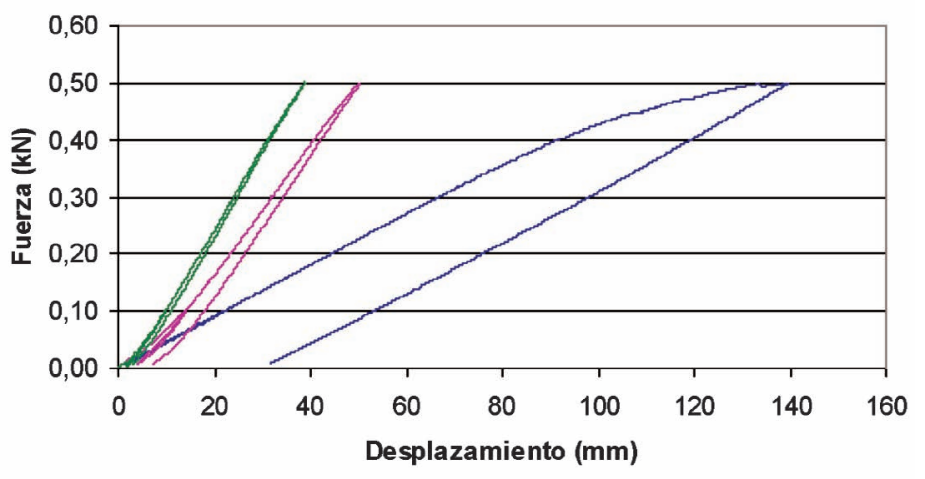

Sistema $1(025 \cdot 1,5) ـ$ Sistema $2(040 \cdot 1,5) —$ Sistema $3(040 \cdot 2,0)$ sobre los 3 sistemas estudiados cuando las cargas se aplican sobre la barandilla principal (punto 1), el rodapié (punto 3) y el extremo superior del poste (punto 4). Los datos de la barandilla intermedia (punto 2) no se reflejan al ser menos desfavorables a los de la barandilla principal.

En las Figuras 6 y 7 se muestran los resultados correspondientes a los ensayos de flecha cuando la carga se aplica sobre el punto central de la barandilla principal (Figura 6) y sobre el punto extremo del poste (Figura 7).

Los resultados del ensayo de resistencia se adjuntan en las Figuras 8 y 9 , cuando la carga se aplica sobre el punto central de la barandilla principal y sobre el extremo volado del poste, respectivamente.

En la figura 10 se muestran los resultados del ensayo de flecha y de resistencia sobre un rodapié.

De los tres sistemas ensayados únicamente el sistema 1 no ha superado el ensayo de carga accidental.

\section{ANÁLISIS Y DISCUSIÓN DE LOS RESULTADOS}

\subsection{Resultados generales}

Los resultados indicados en la Tabla 2 muestran que únicamente el sistema 3 es capaz de superar los requisitos indicados por la norma UNE-EN 13374 para su evaluación experimental. El sistema 1, utilizado de forma habitual en obras, no supera los requisitos de flecha ni de resistencia. El sistema 2, que se utiliza en obras de forma excepcional, no supera el ensayo de resistencia.

\subsection{Requisito de flecha}

En la Figura 6 puede comprobarse que el comportamiento de los 3 sistemas es prácticamente elástico y lineal, siendo el sistema 3 más rígido que el 2 y éste, a su vez, más rígido que el 1 .

El sistema 1 no cumple el requisito de flecha. Cuando se aplica una fuerza de $0,30 \mathrm{kN}$ en el punto central de la barandilla principal, se obtiene un movimiento superior al límite establecido por la norma: $66,67 \mathrm{~mm}$ frente a $55 \mathrm{~mm}$, (Tabla 2).

El movimiento del poste cuando se aplica la carga sobre la barandilla, se puede obtener en la Figura 9, donde para una carga de $0,15 \mathrm{kN}$, se obtiene un movimiento de aproximadamente 11,79 $\mathrm{mm}$. De modo que la barandilla experimenta una flecha de $66,77-11,79=54,98 \mathrm{~mm}$, prácticamente 
Tabla 2

Resultados de los ensayos de flecha y resistencia

\begin{tabular}{|c|c|c|c|c|c|c|c|}
\hline \multirow{2}{*}{ ELEMENTO } & \multirow{2}{*}{ SISTEMA } & \multicolumn{7}{|c|}{ ENSAYO } \\
\cline { 3 - 8 } & & \multicolumn{2}{|c|}{ Flecha } & \multicolumn{4}{|c|}{ Resistencia } \\
\cline { 3 - 8 } & $\mathrm{F}_{\mathrm{T} 1}$ & $\begin{array}{c}\delta \\
(\mathrm{kN})\end{array}$ & $\begin{array}{c}\mathrm{F}_{\mathrm{H} 1} \\
(\mathrm{kN})\end{array}$ & $\begin{array}{c}\mathrm{R}_{\mathrm{u}} \\
(\mathrm{kN})\end{array}$ & $\begin{array}{c}\delta_{\text {máx }} \\
(\mathrm{mm})\end{array}$ & $\begin{array}{c}\delta_{\text {res }} \\
(\mathrm{mm})\end{array}$ \\
\hline \multirow{3}{*}{$\begin{array}{c}\text { Barandilla } \\
\text { principal }\end{array}$} & $\mathrm{S} 1$ & 0,30 & $\mathbf{6 6 , 6 7}$ & 0,50 & $\mathbf{0 , 5 7}$ & 138,24 & $\mathbf{3 0 , 2 7}$ \\
\cline { 2 - 8 } & $\mathrm{S} 2$ & 0,30 & 28,87 & 0,50 & 1,34 & 46,69 & 3,17 \\
\cline { 2 - 8 } & $\mathrm{S} 3$ & 0,30 & 21,66 & 0,50 & 1,94 & 37,20 & 1,20 \\
\hline \multirow{3}{*}{ Poste } & $\mathrm{S} 1$ & 0,30 & 20,98 & 0,50 & 1,08 & 28,62 & 1,60 \\
\cline { 2 - 8 } & $\mathrm{S} 2$ & 0,30 & 19,48 & 0,50 & 0,82 & 34,12 & $\mathbf{6 , 2 5}$ \\
\cline { 2 - 8 } & $\mathrm{S} 3$ & 0,30 & 14,97 & 0,50 & 1,41 & 23,15 & 1,32 \\
\hline Rodapié & $\mathrm{S} 1, \mathrm{~S} 2, \mathrm{~S} 3$ & 0,20 & 13,43 & 0,35 & 0,63 & 30,18 & 2,25 \\
\hline
\end{tabular}

Tabla 3

Resultados de desplazamiento para los tres sistema ensayados.

\begin{tabular}{|c|c|c|c|}
\hline & SISTEMA 1 & SISTEMA 2 & SISTEMA 3 \\
\hline \multirow{2}{*}{ POSTE } & $040 \cdot 1,5$ & $\square 35 \cdot 1,5$ & $040 \cdot 2,0$ \\
& $10,49 \mathrm{~mm}$ & $9,74 \mathrm{~mm}$ & $7,49 \mathrm{~mm}$ \\
\hline \multirow{2}{*}{ BARANDILLA } & $025 \cdot 1,5$ & $040 \cdot 1,5$ & $040 \cdot 2,0$ \\
& $56,18 \mathrm{~mm}$ & $19,13 \mathrm{~mm}$ & $14,17 \mathrm{~mm}$ \\
\hline \multirow{2}{*}{ SISTEMA } & $66,67 \mathrm{~mm}$ & $28,87 \mathrm{~mm}$ & $21,66 \mathrm{~mm}$ \\
\hline
\end{tabular}

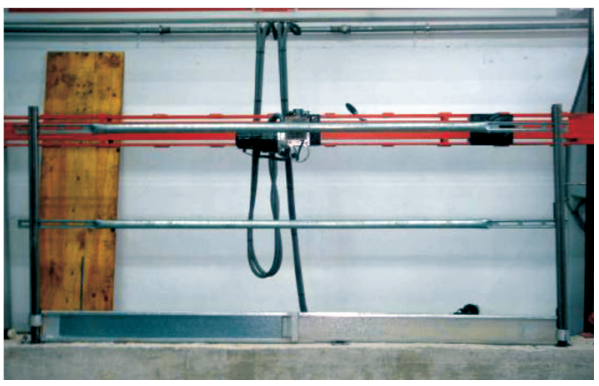

$55 \mathrm{~mm}$. Esto quiere decir que incluso siendo el poste infinitamente rígido (supuesto imposible), la barandilla por sí sola experimenta un movimiento igual al máximo admitido para el sistema, luego el sistema completo ya no es válido.

Por otra parte, el poste experimenta un movimiento de 20,98 mm (Tabla 2) al aplicar la totalidad de la carga sobre él, lo que indica que con una barandilla lo suficientemente rígida, podría superar el ensayo de flecha. En concreto y para superar este ensayo, la barandilla podría desplazarse 55 - 10,49= $44,51 \mathrm{~mm}$.

El rodapié, con un movimiento de 13,43 mm, cumple el requisito de flecha (Tabla 2 ).

Al aumentar la rigidez de la barandilla, el sistema 2 es capaz de cumplir el requisito de flecha (Figura 11). En la Tabla 2 se puede comprobar que la flecha del sistema al aplicar la carga en el centro de la barandilla principal es inferior al límite establecido por
9. Resultados del ensayo de resistencia sobre el punto superior del poste.

10. Resultados del ensayo de flecha $x$ de resistencia sobre el punto medio del rodapié.

11. Ensayo de flecha, aplicando la carga en el centro de la barandilla principal del sistema 2
Ensayo de resistencia postes de acero

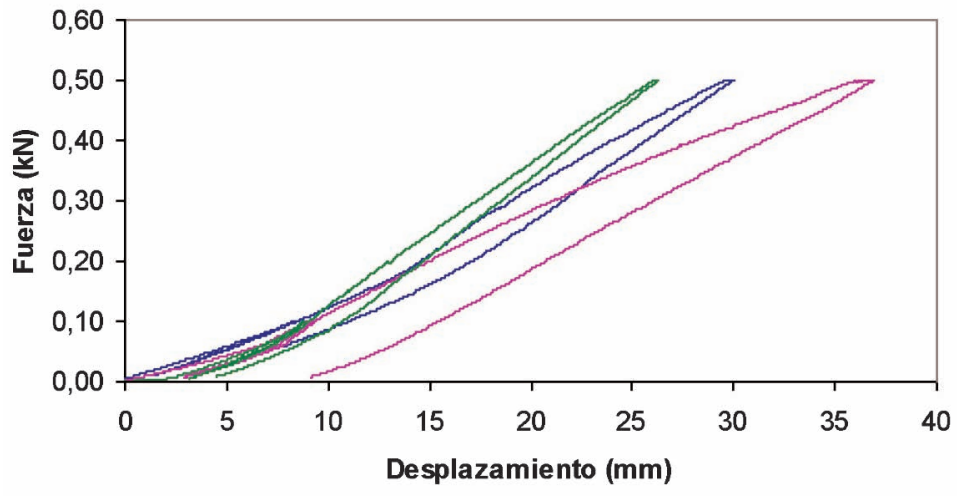

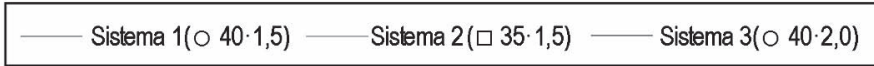

Ensayos de flecha y resistencia rodapié

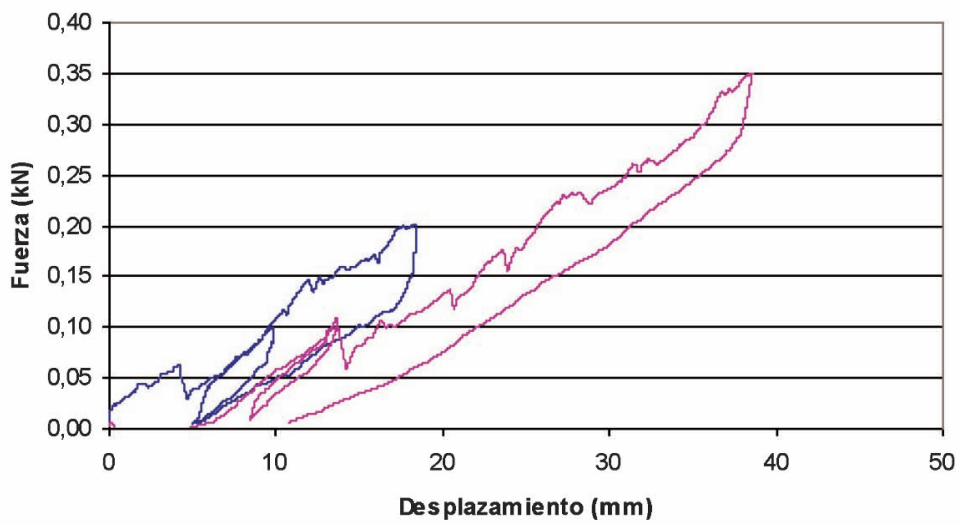


12. Ensayo de resistencia, aplicando la carga sobre el extremo superior del poste del sistema 3 .

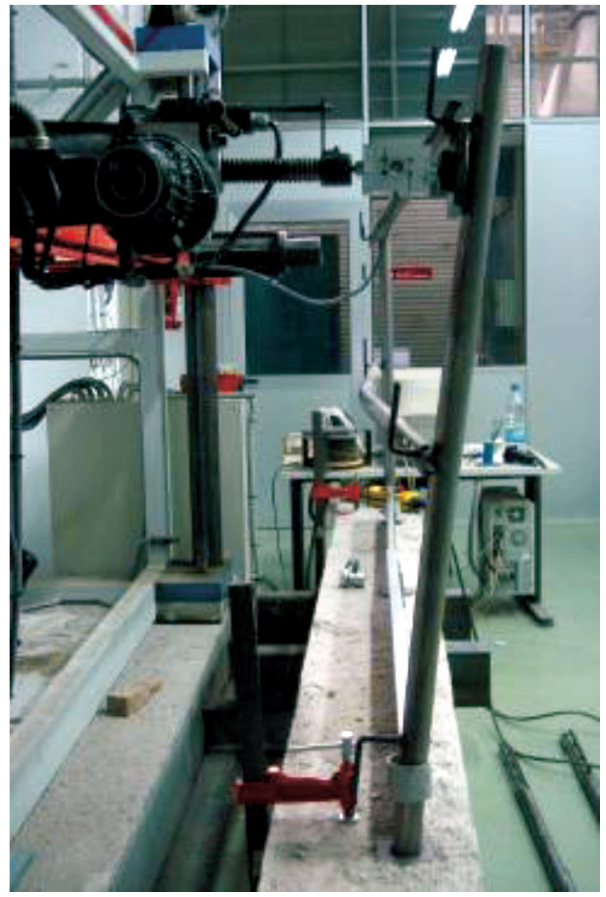

la norma (28,87 $\mathrm{mm}$ frente a $55 \mathrm{~mm})$. Ocurre lo mismo en el sistema 3 cuya flecha máxima del sistema $(21,66 \mathrm{~mm})$ es todavía inferior a la del sistema 2 .

La flecha del poste del sistema 2 se mantiene en valores similares a los del sistema 1.

El aumento de la rigidez de las secciones de barandilla y poste han dado como resultado movimientos significativamente menores en el sistema 3 .

En la Tabla 3 se muestra el movimiento del sistema en los 3 casos estudiados para la carga de $0,30 \mathrm{kN}$ en el centro de la barandilla principal, separando el movimiento correspondiente al poste y a la barandilla.

Puede comprobarse como, para el requisito de flecha, el poste no es determinante y las 3 soluciones anteriores dan valores de flecha para el poste muy similares.

Sin embargo, la diferencia en la flecha de las barandillas es importante. El paso del tubo $40 \cdot 1,5$ al tubo $40 \cdot 2$ supone una disminución significativa de la flecha de la barandilla y la barandilla de $25 \cdot 1,5$, por sí sola, experimenta una flecha superior a la permitida por la norma.

Las gráficas de la Figura 7 muestran en los tres postes un comportamiento inicial anómalo, consecuencia de las deformaciones provocadas en el cartucho de plástico al aplicar la carga, lo que conduce a gráficas no lineales y con rigidización bajo carga. Superada aproximadamente la mitad de la carga de ensayo, el comportamiento es lineal.
La descarga se produce de forma elástica, siguiendo muy aproximadamente la curva correspondiente a la carga. En cualquiera de los postes se obtiene un movimiento inferior al límite permitido por la norma.

\subsection{Requisito de resistencia}

En la Figura 8, se muestra el diagrama fuerzadesplazamiento para una carga aplicada en el punto medio de la barandilla principal.

Se vuelven a poner de manifiesto las distintas rigideces de los tres sistemas. En el sistema 1 el diagrama es lineal hasta aproximadamente una carga de $0,40 \mathrm{kN}$, a partir de ese punto se pierde la linealidad y los movimientos crecen más rápidamente que las fuerzas, perdiendo el sistema rigidez de forma paulatina, como consecuencia de la plastificación que está produciendo en el sistema. Al alcanzar la máxima carga y descargar, la descarga se produce por una recta paralela a la de aplicación de las cargas y queda una flecha residual de aproximadamente $30 \mathrm{~mm}$ $(30,07 \mathrm{~mm})$. En este caso el sistema resiste la máxima carga de ensayo pero no cumple los otros dos requisitos de la norma: la flecha residual es superior al $10 \%$ de la máxima instantánea y la resistencia última $(0,57$ kN) no es superior a 1,2 veces la máxima carga de ensayo, 0,60 kN, (Tabla 2).

El comportamiento de los sistemas 2 y 3 es lineal, no apreciándose prácticamente deformaciones remanentes. Se cumplen los tres puntos indicados por la norma para la superación del requisito de resistencia (Tabla 2).

En la gráfica carga-desplazamiento correspondiente al poste del sistema 1 (Figura 9), de nuevo se vuelve a apreciar un comportamiento anómalo según aumenta la carga, con un incremento de la rigidez del sistema, motivado por las deformaciones entre el cartucho de plástico embebido en la viga y el poste metálico. Cuando finalizan esas deformaciones (aproximadamente para 0,20 kN), el comportamiento corresponde a la rigidez del poste. Se alcanza la máxima carga de ensayo, produciéndose la descarga de forma similar a la carga, lo que significa que las deformaciones producidas en el cartucho de plástico se recuperan y que el comportamiento del poste es elástico. El poste supera el ensayo de resistencia.

Cuando la carga se aplica sobre el poste del sistema 2, a partir de aproximadamente 0,32 kN (Figura 9), se pierde la linealidad y aunque el poste es capaz de resistir la carga máxima de ensayo sin rotura y la carga última es superior al valor especificado, la deformación remanente supera al 10\% de 
Tabla 4

Comparación entre los resultados analíticos y experimentales en la comprobación de flecha

\begin{tabular}{|c|c|c|c|}
\hline \multicolumn{2}{|c|}{} & ANALÍTICO $(\mathrm{mm})$ & EXPERIMENTAL $(\mathrm{mm})$ \\
\hline \multirow{2}{*}{ S 1} & POSTE $\circ 40 \cdot 1,5$ & 7,10 & 10,49 \\
\cline { 2 - 4 } & SISTEMA & $\mathbf{6 0 , 7 0}$ & $\mathbf{6 6 , 6 7}$ \\
\hline \multirow{2}{*}{ S 2} & POSTE $\square 35 \cdot 1,5$ & 6,32 & 9,74 \\
\cline { 2 - 4 } & SISTEMA & 18,54 & 28,87 \\
\hline \multirow{2}{*}{ S 3 } & POSTE O $40 \cdot 2$ & 5,51 & 7,49 \\
\cline { 2 - 4 } & SISTEMA & 15,03 & 21,66 \\
\hline
\end{tabular}

Tabla 5

Comparación entre los resultados analíticos y experimentales en la comprobación de resistencia

\begin{tabular}{|c|c|c|c|c|c|c|}
\hline \multicolumn{2}{|c|}{} & \multicolumn{2}{|c|}{ ANALÍTICO } & \multicolumn{3}{c|}{ EXPERIMENTAL } \\
\cline { 2 - 7 } \multicolumn{2}{|c|}{} & $\mathrm{M}_{\mathrm{Sd}}(\mathrm{kN} \cdot \mathrm{m})$ & $\mathrm{M}_{\mathrm{Rd}}(\mathrm{kN} \cdot \mathrm{m})$ & $\begin{array}{c}\mathrm{F}_{\mathrm{H} 1} \\
(\mathrm{kN})\end{array}$ & $\begin{array}{c}\mathrm{R}_{\mathrm{u}} \\
(\mathrm{kN})\end{array}$ & $\begin{array}{c}\delta_{\text {res }} \\
(\mathrm{mm})\end{array}$ \\
\hline \multirow{3}{*}{ BARANDILLA } & $\mathrm{S} 1$ & 0,27 & $\mathbf{0 , 1 8}$ & 0,50 & $\mathbf{0 , 5 7}$ & $\mathbf{3 0 , 2 7}$ \\
\cline { 2 - 7 } & $\mathrm{S} 2$ & 0,27 & 0,48 & 0,50 & 1,34 & 3,17 \\
\cline { 2 - 8 } & $\mathrm{S} 3$ & 0,27 & 0,62 & 0,50 & 1,94 & 1,20 \\
\hline \multirow{3}{*}{ POSTE } & $\mathrm{S} 1$ & 0,45 & 0,48 & 0,50 & 1,08 & 1,60 \\
\cline { 2 - 8 } & $\mathrm{S} 2$ & 0,45 & 0,54 & 0,50 & 0,82 & $\mathbf{6 , 2 5}$ \\
\cline { 2 - 7 } & $\mathrm{S} 3$ & 0,45 & 0,62 & 0,50 & 1,41 & 1,32 \\
\hline
\end{tabular}

la instantánea y, por tanto, el sistema debe considerarse como no válido (Tabla 2).

En cuanto al poste del sistema 3, se comprueba que de nuevo el comportamiento es elástico y se vuelven a superar los requisitos de la norma (Figura 12).

En la Figura 10 se puede comprobar el enorme movimiento producido en el rodapié cuando se carga hasta $0,10 \mathrm{kN}$ y posteriormente se descarga para obtener la flecha de referencia. La curva cargadesplazamiento, tanto para flecha como para resistencia, muestra el típico aspecto en "diente de sierra" como consecuencia de los deslizamientos producidos en el sistema telescópico del rodapié. Se alcanza la máxima carga de ensayo, y se descarga de forma aproximadamente lineal. En este caso se superan los ensayos de flecha y de resistencia (Tabla 2 y Figura 10).

\subsection{Comparación entre los resultados analíticos y experimentales.}

En la Tabla 4 se muestran los valores de desplazamiento obtenidos experimental y analíticamente por los tres sistemas en el ensayo de flecha.

Para el sistema 1 puede comprobarse que tanto analítica como experimentalmente, se obtiene el mismo resultado: no se supera el requisito de desplazamiento. Los sistemas 2 y 3 superan el requisito de desplazamiento en los dos tipos de evaluación.

Los movimientos obtenidos en la evaluación experimental son mayores que los calculados analíticamente en todos los casos. La razón estriba en que el resultado calculado analíticamente se ha obtenido tomando como modelo de cálculo para el poste una ménsula (figuras 3 y 4). Sin embargo y tal como se muestra en las figuras 7 y 9 el comportamiento del poste no se corresponde al modelo empotrado sino que existen movimientos importantes como consecuencia de las deformaciones del cartucho de plástico en el que se embebe el poste $y$, como consecuencia, se obtienen experimentalmente mayores movimientos que analíticamente. Sería necesario para la evaluación analítica incorporar un modelo que tuviese en cuenta la interacción poste-cartucho de PVC. 
En la Tabla 5 se muestra un resumen de los resultados frente al cálculo a ELU y el ensayo de resistencia para los tres sistemas.

En la columna correspondiente a los resultados analíticos, se indican los valores obtenidos para el momento solicitación $\left(M_{\mathrm{sd}}\right)$ y el momento que es capaz de resistir la sección $\left(M_{R d}\right)$. La comprobación en ELU exige que $M_{R d} \geq M_{S d}$. En la columna correspondiente a los resultados experimentales se indican los valores de la carga de ensayo $\left(\mathrm{F}_{\mathrm{H} 1}\right)$, la resistencia última $\left(R_{\mathrm{u}}\right)$ y la flecha residual $\left(\boldsymbol{\delta}_{\text {res }}\right)$. Los valores marcados en negrilla corresponden a las situaciones en las cuales no se supera la comprobación correspondiente.

Puede comprobarse en el sistema 1 que por ambos procedimientos se obtienen los mismos resultados. El poste cumple a ELU, aunque muy justo $(0,48 \mathrm{kN} \cdot \mathrm{m}$ frente a 0,45 $\mathrm{kN} \cdot \mathrm{m})$, pero la barandilla no es capaz de superar la comprobación a flexión $(0,18 \mathrm{kN} \cdot \mathrm{m}$ frente a $0,27 \mathrm{kN} \cdot \mathrm{m})$.

En el caso de la barandilla, la condición establecida en el cálculo analítico (momento solicitación inferior al momento que resiste la sección) se comprobaría experimentalmente únicamente verificando que es capaz de aguantar la máxima carga de ensayo. Experimentalmente esta condición si se alcanza y son las otras dos condiciones de flecha residual y resistencia última, las que hacen que el ensayo no se supere.

En el sistema 2 puede comprobarse que para la barandilla se obtienen las mismas conclusiones en el cálculo a ELU y en el ensayo de resistencia.

Las discrepancias se generan en la comparación de los resultados para el poste. El poste cumple los cálculos en ELU y, sin embargo, cuando se ensaya no es capaz de superar el ensayo de resistencia porque si bien resiste la máxima carga de ensayo y además su resistencia última es superior a 1,2 veces la carga de ensayo, la flecha residual supera el $10 \%$ de la máxima flecha instantánea.

La discrepancia en los resultados se produce porque los requisitos exigidos por la norma para el análisis experimental son superiores a los exigidos para el cálculo analítico, ya que lo que se exige al cálculo analítico es solamente una de las condiciones exigidas para superar la vía experimental: que se resista la máxima carga de ensayo. Eso es lo que se hace en el cálculo analítico, comprobar que el momento que resiste la sección del elemento es superior al momento producido por la carga de cálculo, pero en el cálculo analítico no se piden las otras dos condiciones exigidas experimentalmente: la resistencia última y la flecha residual, y es precisamente esta última condición de flecha residual la que no ha cumplido el poste.

En el sistema 3, se obtienen los mismos resultados mediante el cálculo en ELU y el ensayo de resistencia.

\section{CONCLUSIONES}

1. La mayoría de los SPPB utilizados habitualmente en obras no cumplen los requisitos exigidos por la norma UNEEN 13374 cuando se evalúan analítica o experimentalmente.

2. El poste de sección tubular cuadrada $35 \cdot 1,5$ no es capaz de superar el ensayo de resistencia de la norma UNE-EN 13374.

3. La evaluación experimental indicada por la norma UNE-EN 13374 es más exigente que la analítica, al indicar en el ensayo de resistencia dos comprobaciones (resistencia última y flecha residual) que analíticamente no se exigen. Precisamente por esta razón el poste indicado en el punto anterior no supera el ensayo de resistencia.

4. Para SPPB con separación entre postes de $2.400 \mathrm{~mm}$, el sistema formado por tubo de acero $40 \cdot 2$ para el poste y la barandilla es capaz de superar satisfactoriamente las exigencias de la norma UNE-EN 13374 tanto por vía analítica como experimental.

5. Combinando los resultados de las Tablas 4 y 5 sistemas formados por barandilla y poste de sección tubular $40 \cdot 1,5$ también serían capaces de superar los requisitos de la norma, analítica y experimentalmente, para SPPB con separaciones de postes de hasta $2.400 \mathrm{~mm}$.

\section{AGRADECIMIENTOS}

Los autores desean agradecer tanto la financiación aportada por el Colegio Oficial de Aparejadores, Arquitectos Técnicos e Ingenieros de Edificación de Madrid para la realización de este trabajo, como la atención recibida desde el Laboratorio de Elementos de Seguridad de AIDICO. 


\section{BIBLIOGRAFÍA}

(1) AENOR-CEN. UNE-EN 13374: "Sistemas provisionales de protección de borde. Especificaciones del producto, métodos de ensayo", Asociación Española de Normalización (AENOR), 2004.

(2) OSHA. "Analysis of Construction Fatalities", The OSHA Data Base 1985-1989. U.S. Department of labor, Washington, DC, 1990.

(3) SESS. Service d'expertise et soutien statistique, IRSST-Sources: Base de données de I'INFOCENTRE de la CSST, mise à jour au 1 mai 1999, 1999.

(4) Ministerio de Trabajo y Asuntos Sociales. Instituto Nacional de Seguridad e Higiene en el Trabajo. «VI Encuesta Nacional de condiciones de trabajo». NIPO: 211-07-057-0. Disponible en: http://www.mtas.es/insht/statistics/viencuesta.pdf [Consulta: 2 junio 2008].

(5) Ministerio de la Presidencia: Real Decreto 1627/1997, de 24 de octubre, por el que se establecen disposiciones mínimas de seguridad y salud en las obras de construcción. Boletín Oficial del Estado, 25 de octubre de 1997, n 256. Anexo IV. Disposiciones de seguridad y salud que deberán aplicarse a las obras. Parte C. Disposiciones mínimas específicas relativas a puestos de trabajo en las obras en el exterior de los locales. 3. Caídas de altura: a).

(6) Ministerio de Trabajo y Asuntos Sociales: IV Convenio General del Sector de la Construcción 2007-2011. Resolución de 1 de agosto de 2007. Boletín Oficial del Estado, 17 de agosto de 2007, n 197. Libro II: Aspectos relativos a la seguridad y salud en el sector de la construcción. Título IV. Disposiciones mínimas de seguridad y salud aplicables a las obras de construcción.

(7) OSHA. Part 1926 Subpart M CFR 1926.500 - Fall Protection for the Construction Industry. Occupational Safety \& Health Administration. US Department of Labor, Washington, DC, 1998.

(8) Québec Safety Code for the Construction Industry. S-2.1, r.6, 2001. Les Publications du Québec, Gouvernement du Québec, Québec, 2001.

(9) OPPBTP. Mémo-practique B1 M 01 94. Les montants de garde-corps provisoires de chantier. Organisme Professionnel de Prévention du Bâtiment et des Travaux Publics. Boulogne-Billancourt, France, 1994.

(10) Jefatura del Estado: Ley 31/1995, de 8 de noviembre, de Prevención de Riesgos Laborales. Boletín Oficial del Estado, 10 de noviembre de 1995, nº 269.

(11) AENOR-CEN. UNE-EN 1263-1: "Redes de seguridad. Parte 1: Requisitos de seguridad, métodos de ensayo", Asociación Española de Normalización (AENOR), 1997.

(12) AENOR-CEN. UNE-EN 1263-2: "Redes de seguridad. Parte 2: Requisitos de seguridad para la instalación de redes de seguridad", Asociación Española de Normalización (AENOR), 1998.

(13) Irles, R.; González, A.; Segovia, E.; Maciá, A.: "Las redes verticales de seguridad en la construcción de edificios. I". Informes de la Construcción, Vol. 53, n 477 (2002), pp. 21-29. doi: 10.3989/ic.2002.v53.i477.

(14) Segovia, E.; Irles, R.; González, A.; Maciá, A.; Pomares, J.: "Las redes verticales de seguridad en la construcción de edificios. II". Informes de la Construcción, Vol. 59, n 505 (2007), pp. 37-51. doi: 10.3989/ic.2007.v59.i505.

(15) García, J.; Irles, R.; Segovia, E.; Pomares, J. C.: "Definición de ensayos a realizar sobre anclajes de seguridad en redes y EPI's". IV Congreso de la Asociación Científico-Técnica del Hormigón Estructural. Valencia. 24 al 27 de noviembre, 2008.

(16) AENOR-CEN. UNE-EN 795: "Protección contra caídas de altura. Dispositivos de anclaje. Requisitos y ensayos", Asociación Española de Normalización (AENOR), 1997.

(17) AENOR-CEN. UNE-EN 795/A1: "Protección contra caídas de altura. Dispositivos de anclaje. Requisitos y ensayos", Asociación Española de Normalización (AENOR), 2001.

(18) eLCOSH. Guiones orientadores para la protección anticaída de alturas. Biblioteca Electrónica de Salud y Seguridad Ocupacional en la Construcción. Disponible en: http://www. cdc.gov/elcosh/docs/d0100/d000052/d000052-s.html [Consulta: 10 marzo 2008].

(19) OPPBTP. Mémo-practique B1 F 01 93. "Protections Collectives pour empecher les chutes d'hauteur dans le bâtiment et les travaux publics" Organisme Professionnel de Prévention du Bâtiment et des Travaux Publics. France, 1993.

(20) ASTM E 985-87. Standard Specification for Permanent Metal Railing Systems and Rail for Buildings. American Society for Testing and Materials, Philadelphia, PA, 1987.

(21) AENOR-CEN. ENV 1993-1-1. "Eurocódigo 3: Proyecto de estructuras de acero. Parte 1-1: Reglas generales y reglas para edificación", Asociación Española de Normalización (AENOR), 1993. 